\title{
Correction to: Mitochondrial DNA Phylogeny of M Haplogroup in Indian Population
}

\section{Correction to:}

Chapter 2 in: Anthropological Survey of India, Genomic Diversity in People of India, https://doi.org/10.1007/978-981-16-0163-7_2

This chapter was inadvertently published with an incorrect title, which has now been corrected to "Mitochondrial DNA Phylogeny of M Haplogroup in Indian Population". 